\title{
Cholangiocarcinoma cell line TK may be useful for the pharmacokinetic study of the chemotherapeutic agent gemcitabine
}

\author{
MINORI KAMADA ${ }^{1 *}$, KOHEI AKIYOSHI ${ }^{2}$, NOBUTAKE AKIYAMA ${ }^{3}$, NAOTAKE FUNAMIZU ${ }^{4}$, \\ MICHIKO WATANABE ${ }^{5}$, KOUKI FUJIOKA ${ }^{2}$, KEI-ICHI IKEDA ${ }^{2}$ and YOSHINOBU MANOME ${ }^{2}$ \\ ${ }^{1}$ Institute of DNA Medicine; Departments of ${ }^{2}$ Molecular Cell Biology, ${ }^{3}$ Molecular Immunology, \\ Research Center for Medical Sciences; ${ }^{4}$ Department of Surgery, Jikei University School \\ of Medicine, Tokyo; ${ }^{5}$ Institute of DNA Sciences, Yokohama, Japan
}

Received March 4, 2014; Accepted April 25, 2014

DOI: 10.3892/or.2014.3227

\begin{abstract}
Cholangiocarcinoma is a disease with a poor prognosis. A human cholangiocarcinoma cell line, TK, was previously established to enable further understanding of the disease. We conducted this investigation to determine whether or not the TK line is useful for pharmacokinetic study of the chemotherapeutic agent gemcitabine (GEM). Along with the BXPC3 human pancreatic adenocarcinoma cell line, the sensitivity to and effects on the TK cell line of GEM were compared. The influence of deoxycytidine kinase (dCK) transduction was also comparatively investigated. The effects of GEM in terms of drug sensitivity of the TK cell line, cell cycle and levels of transcripts of key enzymes were comparable to the BXPC3 cell line. Responses to the drug were similar in both cell lines. In contrast to pancreatic carcinoma, cell lines for research on cholangiocarcinoma have been limited. This study suggests the application of the TK cell line to the pharmacokinetic study of the chemosensitization of therapeutic drugs, such as GEM.
\end{abstract}

\section{Introduction}

Cholangiocarcinoma is one of the most intractable malignancies in the human body. The disease was recently highlighted in Japan, since 11 patients presented with intrahepatic or extrahepatic cholangiocarcinoma among 62 workers who had been employed in the offset color proof-printing room or in the front room of a printing company for at least 1 year between 1991 and 2006. The incidence suggests that cholangiocarcinoma might be a new occupation-related disease. Long-term expo-

Correspondence to: Dr Minori Kamada, Institute of DNA Medicine, Jikei University of School of Medicine, 3-25-8 Nishishinbashi, Minato-ku, Tokyo, 105-8461, Japan

E-mail:mkamada@jikei.ac.jp

Key words: cholangiocarcinoma, chemosensitivity, TK cell line, gemcitabine sure to chemicals, such as 1,2-dichloropropane and/or possibly dichloromethane, were attributable to the etiology (1).

The prognosis of the disease is unfavorable. The 5-year survival of newly diagnosed patients is less than $15 \%$ (2). Surgical removal is the only curable treatment for the disease. However, since the disease initially lacks subjective symptoms and many patients are diagnosed at the advanced stage, only less than a third of all patients are indicated for radical resection (3). Furthermore, even when the affected region is surgically removed, the disease frequently recurs and thus the outcome is not satisfactory. Therefore, intensive chemotherapy is mandatory as an adjunctive treatment of the disease. However, the response rate of systemic chemotherapy is only $\sim 25 \%$ and it is critical to find new treatment strategies for these tumors (3).

Gemcitabine (GEM) is the most potent chemotherapeutic agent for cholangiocarcinoma (4) and from the results of the ABC-02 trial, its combination with cisplatin has become the standard treatment for the disease (5). Nevertheless, the median survival time is less than 12 months in patients at an advanced stage. Studies regarding other approaches, such as its combination with molecular targeting drugs (2) or methods increasing cellular sensitivity to the drugs, are in progress (4).

In order to develop these therapies, cell biological techniques have been utilized. The in vitro study method with corresponding cell lines is particularly useful for evaluating sensitivity to chemotherapeutic agents and testing for the improvement of efficacy. Several cholangiocarcinoma cell lines have been reported (6-10). However, the number and access to these lines is limited and more importantly, detailed information and their documented characteristics are insufficient.

Previously, we established a cholangiocarcinoma cell line and designated it as TK. The cell line was derived from the ascites of a 78-year-old female cholangiocarcinoma patient; it produces carbohydrate antigen (CA)19-9, CA50 and carcinoembryonic antigen (CEA) (11). The cell line also morphologically forms a characteristic duct-like edifice covered by structured microvilli when cultured three-dimensionally, hence the cell line is expected to be usable as a model for in vivo study of 
cholangiocarcinoma (12). The cell line is also implantable and forms a tumor in the nude mouse, and therefore, can be used as a xenograft animal model.

In the present study, the efficacy of GEM on the cell line was investigated. To develop highly effective chemotherapy, cytotoxicities to the drug and transcripts of enzymes relating to GEM incorporation and excretion in the cell line were compared to a pancreatic carcinoma cell line. Pancreatic carcinoma is similarly treated by GEM. By means of a comparative investigation, we attempted to determine whether or not the TK cell line can be used for further studies.

\section{Materials and methods}

Cell lines. Establishment and the characteristics of the TK human cholangiocarcinoma cell line were previously described (11). The TK cell line was cultured with RPMI-1640 (Gibco Life Technologies, Carlsbad, CA, USA) supplemented with $15 \%$ fetal bovine serum (FBS), $2 \mathrm{mM}$ glutamine and $1 \mathrm{mM}$ sodium pyruvate. The human pancreatic adenocarcinoma cell line BXPC 3 was obtained from the American Type Culture Collection (Manassas, VA, USA). BXPC3 was grown in RPMI-1640 containing 10\% FBS, 2 mM glutamine and $1 \mathrm{mM}$ sodium pyruvate. Both cell lines were maintained as monolayer cultures at $37^{\circ} \mathrm{C}$ in a $5 \% \mathrm{CO}_{2}$ atmosphere. Cells were harvested with $0.05 \%$ trypsin and $0.02 \%$ EDTA in Dulbecco's phosphate-buffered saline (PBS).

Cell growth. Growth of the cell line was assessed in 6-cm culture dishes. The cells were seeded at the density of $1.4 \times 10^{4}$ cells per dish, and the cell growth was determined by counting the cell number.

Drug. Gemcitabine (GEM; difluorodeoxycytidine, dFdC) was purchased from Wako (Osaka, Japan). GEM was dissolved in saline, sterilized by $0.22-\mu \mathrm{m}$ filtration, and stored at $-20^{\circ} \mathrm{C}$ until use.

Cytotoxic assay. Cells were seeded in 96-well sterile plates (Corning, Tokyo, Japan) at the density of $5 \times 10^{3}$ cells per well. After $24 \mathrm{~h}$, the cells were treated with stepwise dilutions of GEM and incubated for $120 \mathrm{~h}$. To assess the cell viability, the cells were fixed in $5.4 \%$ glutaraldehyde for $15 \mathrm{~min}$ at room temperature and stained with $50 \mu \mathrm{l}$ of $0.05 \%$ methylene blue for $15 \mathrm{~min}$. The dye was eluted with $0.4 \mathrm{~N} \mathrm{HCl}$ for $15 \mathrm{~min}$, and the absorbance was measured in a microplate reader (model $680 \mathrm{XR}$, Bio-Rad, Tokyo, Japan) at $595 \mathrm{~nm}$ (13). Sensitivity to the agent was evaluated by calculation of the $50 \%$ inhibitory concentration $\left(\mathrm{IC}_{50}\right)$ of the drug.

Cell cycle analysis. The cells were dispersed and attached to the bottom of the culture flasks before use. Subsequently, the cells were exposed to GEM at the dose of the $\mathrm{IC}_{50}$ values for the respective cells. After 48 and $96 \mathrm{~h}$, the cells were dispersed with trypsin, washed with PBS and fixed with $75 \%$ ethanol. They were stained with $50 \mu \mathrm{g} / \mathrm{ml}$ propidium iodide (PI) in PBS with $180 \mathrm{U}$ of RNase A for 30 min (14). The cell cycle populations were determined by a flow cytometer (FACSCalibur; Becton Dickinson Japan, Tokyo, Japan) and analyzed by ModFit Lt software (Becton Dickinson).
Table I. Primers used for RT-PCR.

\begin{tabular}{ll}
\hline Gene & \multicolumn{1}{c}{ Primers } \\
\hline dCK & F: ggaagtggttcetgaacctgttg \\
& R: ctctgcatcttgagcttgcc \\
CDA & F: gaagcgtcctgcctgca \\
& R: ctggaccgtcatgacaatatacg \\
GAPDH & F: gaaggtgaaggtcggagtc \\
& R: gaagatggtgatgggattc \\
\hline
\end{tabular}

F, forward; R, reverse

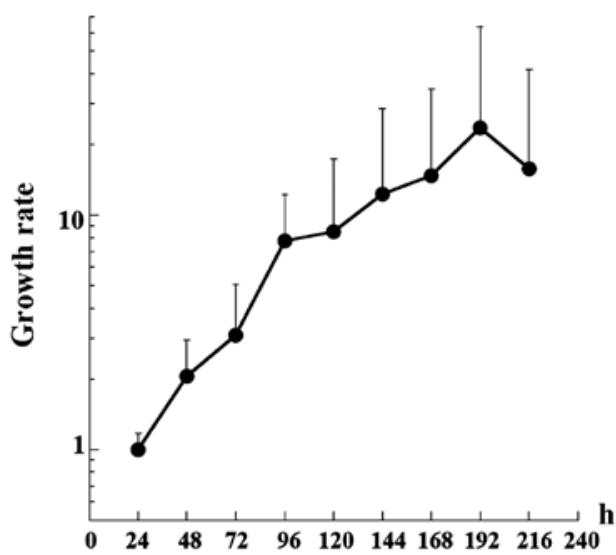

Figure 1. Growth of the TK cell line. The cells were seeded at a density of $1.4 \times 10^{4}$ cells per 6 -cm dish in duplicate. Cell growth was determined by counting the cell number. The doubling time of the cells was $36.9 \mathrm{~h} . \mathrm{n}=8$; bars, SD.

Quantification of RNA transcripts. The cellular RNAs of TK and BXPC 3 cells were extracted by the acid guanidium-phenolchloroform (AGPC) method (RNAzol B; Tell Test, Friendswoods, TX, USA). The RNAs were treated with RNase inhibitor and RNase-free recombinant DNase I (Takara Bio, Otsu, Japan) for $20 \mathrm{~min}$, and the resulting DNA-free RNAs were reversely transcribed using Prime Script RT Master Mix (Takara Bio). For quantification of the RNAs of deoxycytidine kinase (dCK), cytidine deaminase (CDA) and deoxycytidine monophosphate (dCMP) deaminase, semi-quantitative polymerase chain reactions were performed at $95^{\circ} \mathrm{C}$ for $30 \mathrm{sec}$ followed by 40 cycles at $95^{\circ} \mathrm{C}$ for $5 \mathrm{sec}$ and then at $60^{\circ} \mathrm{C}$ for $31 \mathrm{sec}$ (SYBR-Premix Ex Taq II; Takara Bio). Primers used for the study are shown in Table I. Signals were detected using the ABI 7300 real-time PCR system (Applied Biosystems, Life Technologies, Carlsbad, CA, USA) Specificities of the reaction were confirmed with melting curves. The expression level was compared by relative quantification $(\Delta \Delta \mathrm{Ct})(15)$.

Adenoviral infection. Constructs of the adenoviral vectors encoding dCK (Ad-dCK) and green fluorescent protein (GFP) (Ad-GFP) were described previously $(13,16)$. These viral vectors were isolated from single plaques, expanded in human embryonic kidney 293 cells and purified 


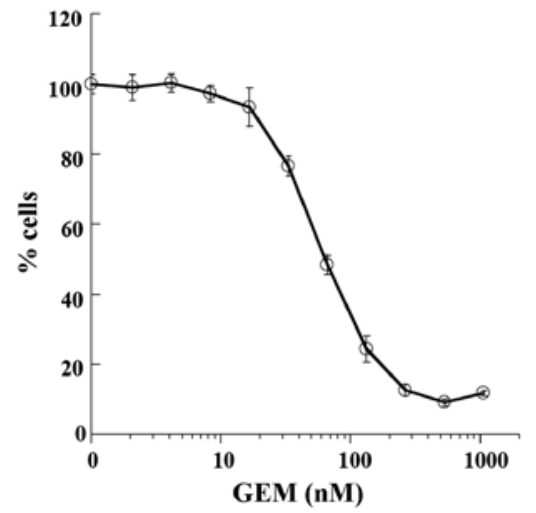

Figure 2. Sensitivity of the TK cell line to GEM. The cells were seeded in 96-well sterile plates at $5 \times 10^{3}$ cells per well. After $24 \mathrm{~h}$, the cells were treated with stepwise dilutions of GEM and incubated for $120 \mathrm{~h}$. Cell viability was assessed by staining the cells with methylene blue and the sensitivity was determined from the $\mathrm{IC}_{50}$ value. $\mathrm{n}=8$; bars, $\mathrm{SD}$.

by double Cs centrifugation (17). The TK and BXPC3 cells were infected with the Ad-dCK or control Ad-GFP at several different multiplicities of infection (MOIs).

Cell growth inhibition assay. After $24 \mathrm{~h}$ of adenoviral infection, the cells were dispersed, seeded in 96-well plates at the density of $1.2 \times 10^{2}$ cells per well, and incubated for $24 \mathrm{~h}$. Cells in each group were exposed to GEM at concentrations of 0 or the dose of the respective $\mathrm{IC}_{50}$ for $120 \mathrm{~h}$ and the cytotoxicity was measured. The effect of dCK transduction on GEM treatment was evaluated by inhibition divided by mock treatment.

Statistical analysis. Statistical analysis was performed by the two-sample t-test.

\section{Results}

Cell growth of the TK cell line. The manner of TK cell growth was examined first. The growth curve of the TK cells is shown in Fig. 1. The doubling time of the cells was $36.9 \mathrm{~h}$, which was slightly longer than that of the earlier passage cells (29 h) (11). The saturation density of the cells was $2.7 \times 10^{5} / \mathrm{cm}^{2}$.

Sensitivity of the TK cell line to GEM. Since GEM is a standard therapeutic agent, sensitivity of the TK cells to the drug was determined (Fig. 2). The GEM $\mathrm{IC}_{50}$ value of the TK cells was $66.22 \mathrm{nM}$ and the value was comparable to that of the pancreatic BXPC3 cells (47.10 nM).

Cell cycle analysis of the TK cells exposed to GEM. To analyze the mechanism of action on these cells, cell cycle populations of both the TK and pancreatic BXPC cell lines were analyzed (Fig. 3). Forty-eight and $96 \mathrm{~h}$ after treatment with GEM, the cells were fixed and the cell populations in each cell cycle were analyzed. At $48 \mathrm{~h}$, the $\mathrm{S}$ phase population was increased in both the TK and BXPC cell lines. An apoptotic sub-G1 peak was observed only in the TK cells, but this peak subsequently also appeared in the BXPC cells at $96 \mathrm{~h}$ after treatment. The overall effects of GEM on the cell cycle of both cell lines were similar.

Quantification of transcripts of deoxycytidine kinase (dCK) and cytidine deaminase (CDA). GEM is a prodrug and confers toxicity only after it is incorporated into DNA. When taken up by cells, the agent is metabolized by cellular enzymes, among which the activities of deoxycytidine kinase (dCK) and cytidine deaminase (CDA) are the major contributors to chemosensitivity. The quantities of $\mathrm{dCK}$ and CDA in the TK and BXPC3 cells were measured (Fig. 4). When compared,
A

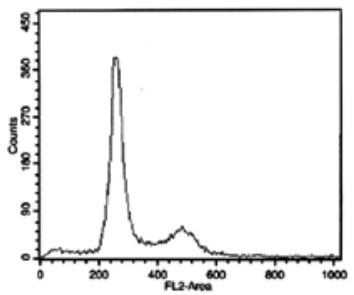

$0 \mathrm{~h}$

B

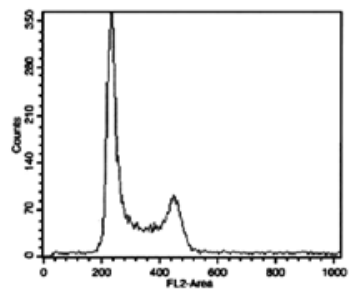

$0 \mathrm{~h}$

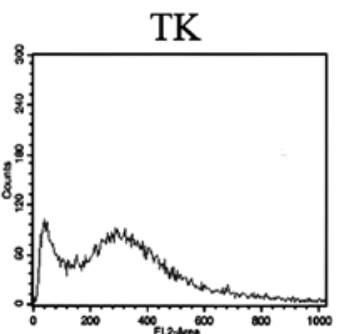

$48 \mathrm{~h}$

BxPC-3

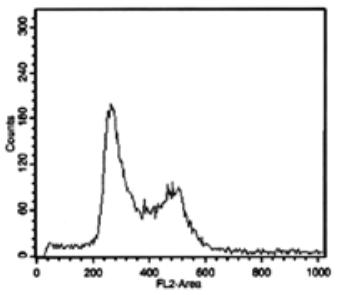

$48 \mathrm{~h}$

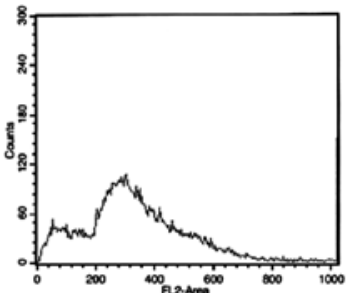

$96 \mathrm{~h}$

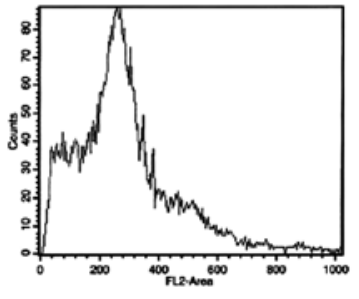

$96 \mathrm{~h}$

Figure 3. Effects of GEM on cell cycle populations at the dose of the $\mathrm{IC}_{50}$ value in each cell line. Cell cycle populations of the (A) TK and (B) BXPC3 cell lines were determined using flow cytometry. At $48 \mathrm{~h}$, the $\mathrm{S}$ phase population increased in both the TK and BXPC3 cell lines. An apoptotic sub-G1 peak was first observed in the TK cells, but this peak also subsequently appeared in the BXPC3 cells. 


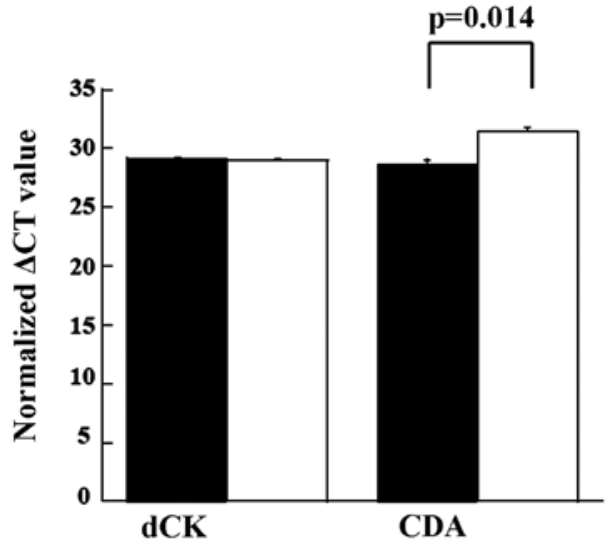

Figure 4. Levels of dCK and CDA transcripts in the TK and BXPC3 cell lines. Quantities of CDA and dCK mRNA in the TK (घ) and BXPC3 () cell lines were evaluated by semi-quantitative PCR. Normalized $\Delta \mathrm{Ct}$ was calculated as 40 - (dCK or CDA Ct - GAPDH Ct). Values are means \pm SD. $n=3$; bars, SD.

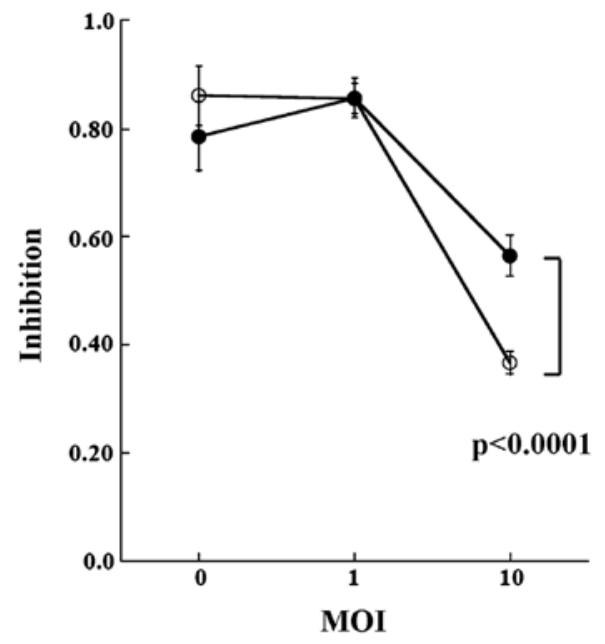

Figure 5. Effect of forced expression of dCK on sensitivity to GEM. The TK (•) and BXPC3 (O) cells were infected with Ad-dCK at several different multiplicities of infection (MOIs). At a viral infection of MOI 0 and 1 , forced expression of dCK did not alter sensitivity to the drug in both cell lines. Meanwhile at a viral infection of MOI 10, forced expression of dCK conferred sensitivity to GEM. $n=7$; bars, $\mathrm{SD}$.

levels of the dCK transcript were almost equal. However, the level of CDA in the TK cell line was lower than that in the BXPC cell line $(\mathrm{P}<0.02)(\Delta \Delta \mathrm{Ct} \mathrm{TK}, 28.6$ vs. BXPC3, 31.4). Transcripts of dCMP deaminase, which also inactivate dFdCMP, were almost equal in both cell lines $(\Delta \Delta \mathrm{Ct} \mathrm{TK}, 31.9$ vs. BXPC3, 32.2).

Sensitization of the cell lines by forced expression of the $d C K$ enzyme. In the pancreatic tumor cells, an increase in $\mathrm{dCK}$ improved the efficacy of GEM (18). The effects of transduction of dCK on sensitivity to GEM were measured (Fig. 5). At viral infection of MOI 0 and 1, overexpression of dCK did not alter the sensitivity to the drug in either cell line. When MOI was increased to 10 , dCK conferred sensitivity to GEM. While both TK and BXPC 3 cells were sensitized by the infection, the effect was marginally higher in the BXPC 3 cells. Transduction of the control GFP did not influence the sensitivities (Fig. 6).

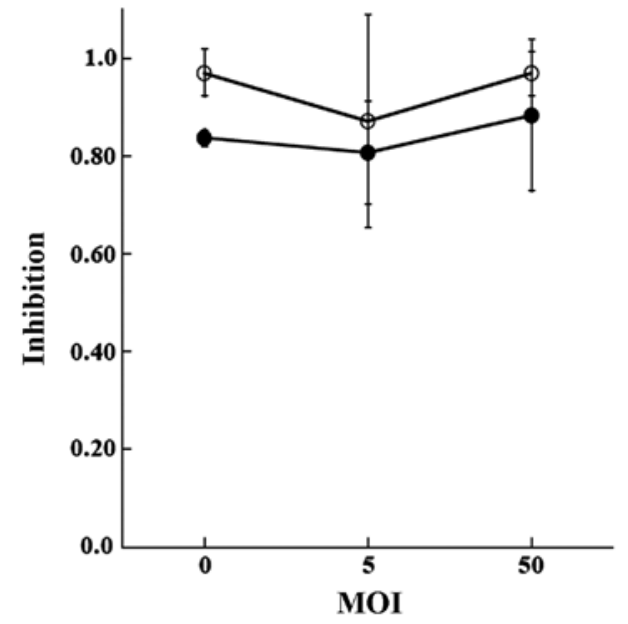

Figure 6. Effect of forced expression of control GFP on sensitivity to GEM. The TK $(\bullet)$ and BXPC3 (O) cells were infected with control Ad-GFP at several different MOIs. Transduction of control GFP did not alter the sensitivities. $\mathrm{n}=7$; bars, $\mathrm{SD}$.

\section{Discussion}

In the present study, the pharmacological response to GEM of the cholangiocarcinoma TK cell line was evaluated. GEM is a representative chemotherapeutic agent used to treat pancreatic carcinoma. The drug is also used to treat cholangiocarcinoma as the agent of first choice. Since GEM has a potent cytotoxic effect and its mechanism of action is relatively well understood, methods to increase its efficacy have been extensively studied. TK and BXPC3 cells were used to compare the effects of GEM on the cell cycle, drug sensitivity, and levels of transcripts of key enzymes. In addition, the effects of dCK transduction were addressed.

Cholangiocarcinoma is a refractory disease. More than half of all patients are inoperable when diagnosed (19). Unresectable or metastatic lesions are treated with chemotherapeutic agents (2). However, the effect has been limited and in this situation, the development of more effective adjuvant therapy is needed $(3,4)$.

GEM (dFdC) is an analog of deoxycytidine and when entering target cells, the drug is phosphorylated to gemcitabine monophosphate (dFdCMP), gemcitabine diphosphate $(\mathrm{dFdCDP})$, and then gemcitabine triphosphate (dFdCTP) by corresponding enzymes. After conversion, dFdCTP is incorporated into DNA by competing with deoxycytidine triphosphate (dCTP). When integrated into DNA, it terminates further chain elongation and causes apoptosis (4). In this sequence, phosphorylation of GEM to dFdCMP by deoxycytidine kinase (dCK) is a rate-limiting reaction. Furthermore, cytidine deaminase (CDA) abolishes the cytotoxicity by deaminating GEM (Fig. 7). GEM and dFdCMP are deaminated by cytidine deaminase (CDA) and dCMP deaminase, and become the inactive products, $2^{\prime}, 2^{\prime}$-difluorodeoxyuridine (dFdU) and 2',2'-difluorodeoxyuridine monophosphate (dFdUMP).

Multiple mechanisms potentiate the activity of GEM both by increased formation of active $\mathrm{dFdCDP}$ and $\mathrm{dFdCTP}$, and decreased elimination of GEM. For example, dFdCDP itself inhibits ribonucleotide reductase (RR) and depletes the deoxyribonucleotide pool that is available for DNA synthesis and 


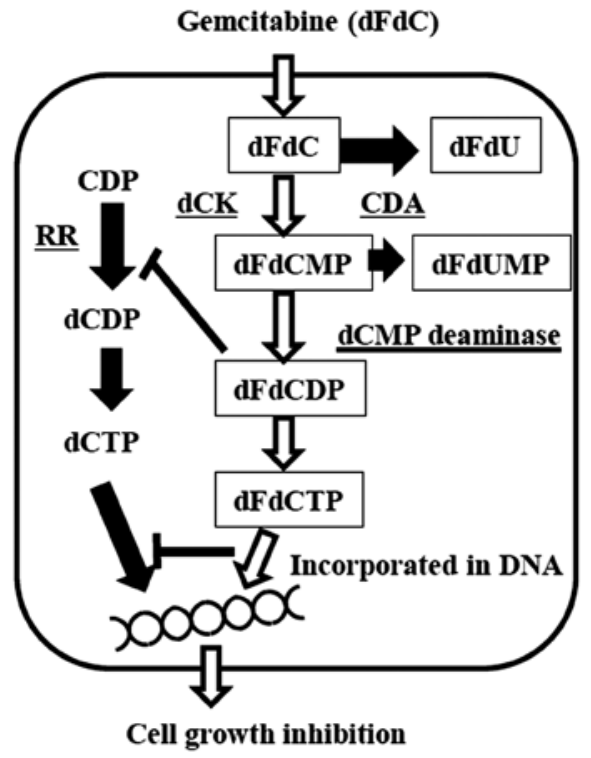

Figure 7. Pathway of GEM metabolism. The schema demonstrates the compendious pathway of GEM metabolism.

repair. A decreased concentration of dCTP activates dCK, which accelerates the phosphorylation of GEM. Furthermore, a decreased concentration of intracellular dCTP inhibits dCMP deaminase and increases the concentration of dFdCTP (20).

The incidence of cholangiocarcinoma differs in each region and country, but overall, this disease accounts for only $\sim 3 \%$ of gastrointestinal system malignancies. The low incidence of the disease, the diversity of the patients and tumor progression affect clinical trial enrollments and retard the development of effective treatments for cholangiocarcinoma (2).

Methods to increase the sensitivity to GEM have been investigated using pancreatic carcinoma cell lines. Funamizu et al demonstrated the effects of transduction of $\mathrm{dCK}$ in activating GEM and tetrahydrouridine (THU), which inhibits the action of CDA in causing GEM resistance $(15,18)$. Nakahira et al reported that inhibition of the ribonucleotide reductase M1 subunit (RRM1) of a GEM-resistant cell line by siRNA improved the effect of GEM (21).

Ohhashi et al demonstrated that inhibition of dCK by siRNA decreased the sensitivity to GEM, and the inhibition of RRM1 and RRM2 by siRNA increased the sensitivity to GEM (22). Duxbury et al also demonstrated that systemic administration of siRNA for RRM2 decreased the expression of RRM2 in an implanted tumor of pancreatic adenocarcinoma and by combination with GEM treatment, inhibited tumor growth, increased apoptotic cells and the inhibition of metastasis using a nude mouse xenograft model (23).

In contrast to pancreatic carcinoma, only a small number of studies on cholangiocarcinoma have been reported and this might be due to the limited number of suitable cell lines. Ohtaka et al demonstrated that inhibition of RRM1 by siRNA increased sensitivity to GEM and induced apoptosis in a gallbladder carcinoma cell line (4). Faris and Zhu investigated the combination of GEM and a molecular targeting agent (2). Other studies aimed to explore the factors for predicting the effect of gemcitabine-based chemotherapies $(3,5,24,25)$.
In the present study, we attempted to demonstrate that the TK cell line might be useful for further studies. Transcripts of $\mathrm{dCK}$ and CDA in the TK and pancreatic BXPC3 cell lines were compared in the study and the level of dCK transcript was comparable in both cell lines. The sensitivity of TK cells to GEM was almost equal to that of the BXPC 3 cell line. Similar to pancreatic adenocarcinoma, patients are treated by GEM and the sensitivity as well as the localization and blood supply of cholangiocarcinoma would provide an adequate justification for using GEM for the treatment of cholangiocarcinoma.

In pancreatic carcinoma, levels of dCK expression determine the prognosis of patients (26) and an increase in $\mathrm{dCK}$ might directly improve the sensitivity to GEM $(18,27)$. Our result demonstrated that the level of the dCK transcript in TK cells was almost the same as that in the BXPC3 cells (Fig. 4) and adenoviral transduction of $\mathrm{dCK}$ was able to increase the sensitivity to the drug (Fig. 5)

Alternatively, CDA abolishes the efficacy of GEM by deamination of the agent. The level of transcripts of CDA was lower in the TK cell line than in the BXPC cell line. Higher sensitivity due to adenoviral transduction of $\mathrm{dCK}$ was expected in the TK cell line. However, our result demonstrated that BXPC3 cells were more sensitized by the procedure. The result itself might be controversial, but many factors, such as RR subunit M1, RR subunit M2, the RR subunit p53R2 gene, and the human equilibrative nucleotide transporter 1 (hENT1) are also important for GEM sensitivity $(3-5,21,24,25,28)$.

In addition, human equilibrative nucleotide transporter 2 (hENT2), human concentrative nucleotide transporter 1 (hCNT1), and human concentrative nucleotide transporter 3 (hCNT3) are responsible for the uptake of GEM, and multidrug resistance-associated protein 7 (MRP7) is related to excretion. All of these factors are associated with the sensitivity of cells to GEM. To understand the mechanisms of these actions, further study is required using clinical samples and/or more cell lines (3).

The effects of GEM on cholangiocarcinoma have been addressed using the TK cell line. Hence, this cell line may have a role for further investigation of GEM sensitivity in relation to cholangiocarcinoma chemotherapy.

\section{Acknowledgements}

The authors thank Mr. Chiaki Kuriyama, Ms. Nanami Takatsuki, Airi Kugisaki, Keiko Tomaru and Mayumi Nomura of the Jikei University School of Medicine for their expert technical assistance.

\section{References}

1. Kumagai S, Kurumatani N, Arimoto A and Ichihara G: Cholangiocarcinoma among offset colour proof-printing workers exposed to 1,2-dichloropropane and/or dichloromethane. Occup Environ Med 70: 508-510, 2013.

2. Faris JE and Zhu AX: Targeted therapy for biliary tract cancers J Hepatobiliary Pancreat Sci 19: 326-336, 2012.

3. Sato J, Kimura T, Saito T, et al: Gene expression analysis for predicting gemcitabine resistance in human cholangiocarcinoma. J Hepatobiliary Pancreat Sci 18: 700-711, 2011.

4. Ohtaka K, Kohya N, Sato K, Kitajima Y, Ide T, Mitsuno M and Miyazaki K: Ribonucleotide reductase subunit M1 is a possible chemoresistance marker to gemcitabine in biliary tract carcinoma. Oncol Rep 20: 279-286, 2008. 
5. Borbath I, Verbrugghe L, Lai R, Gigot JF, Humblet Y, Piessevaux $\mathrm{H}$ and Sempoux C: Human equilibrative nucleoside transporter 1 (hENT1) expression is a potential predictive tool for response to gemcitabine in patients with advanced cholangiocarcinoma. Eur J Cancer 48: 990-996, 2012.

6. Meng F, Henson R, Lang M, et al: Involvement of human micro-RNA in growth and response to chemotherapy in human cholangiocarcinoma cell lines. Gastroenterology 130: 2113-2129, 2006.

7. Pignochino Y, Sarotto I, Peraldo-Neia C, et al: Targeting EGFR/HER2 pathways enhances the antiproliferative effect of gemcitabine in biliary tract and gallbladder carcinomas. BMC Cancer 10: 631, 2010.

8. Saito S, Ghosh M, Morita K, Hirano T, Miwa M and Todoroki T: The genetic differences between gallbladder and bile duct cancer cell lines. Oncol Rep 16: 949-956, 2006.

9. Selaru FM, Olaru AV, Kan T, et al: MicroRNA-21 is overexpressed in human cholangiocarcinoma and regulates programmed cell death 4 and tissue inhibitor of metalloproteinase 3. Hepatology 49: 1595-1601, 2009.

10. Mott JL, Kobayashi S, Bronk SF and Gores GJ: mir-29 regulates Mcl-1 protein expression and apoptosis. Oncogene 26: 6133-6140, 2007.

11. Watanabe M, Chigusa M, Takahashi H, Nakamura J, Tanaka $H$ and Ohno T: High level of CA19-9, CA50, and CEA-producible human cholangiocarcinoma cell line changes in the secretion ratios in vitro or in vivo. In Vitro Cell Dev Biol Anim 36 104-109, 2000.

12. Akiyoshi K, Kamada M, Akiyama N, et al: Morphological study of cholangiocarcinoma cell line, TK with three-dimensional cel culture. Mol Med Rep 9: 1359-1364, 2014.

13. Manome Y, Wen PY, Dong Y, Tanaka T, Mitchell BS, Kufe DW and Fine HA: Viral vector transduction of the human deoxycytidine kinase cDNA sensitizes glioma cells to the cytotoxic effects of cytosine arabinoside in vitro and in vivo. Nat Med 2: 567-573, 1996.

14. Kamada M, Ikeda K, Fujioka K, et al: Expression of mRNAs of urocortin and corticotropin-releasing factor receptors in malignant glioma cell lines. Anticancer Res 32: 5299-5307, 2012 .

15. Funamizu N, Lacy CR, Fujita K, Furukawa K, Misawa T, Yanaga K and Manome Y: Tetrahydrouridine inhibits cell proliferation through cell cycle regulation regardless of cytidine deaminase expression levels. PLoS One 7: e37424, 2012.

16. Suzuki R, Kojima H, Moriyama $\mathrm{H}$ and Manome $\mathrm{Y}$ : Utilization of caspase-14 promoter for selective transgene expression in squamous layers of cholesteatoma in the middle ear. J Intl Adv Otol 8: 21-29, 2012.

17. Manome Y, Wen PY, Chen L, et al: Gene therapy for malignant gliomas using replication incompetent retroviral and adenovira vectors encoding the cytochrome P450 2B1 gene together with cyclophosphamide. Gene Ther 3: 513-520, 1996.
18. Funamizu N, Okamoto A, Kamata Y, et al: Is the resistance of gemcitabine for pancreatic cancer settled only by overexpression of deoxycytidine kinase? Oncol Rep 23: 471-475, 2010.

19. Marsh Rde W, Alonzo M, Bajaj S, et al: Comprehensive review of the diagnosis and treatment of biliary tract cancer 2012. Part I: diagnosis-clinical staging and pathology. J Surg Oncol 106: 332-338, 2012.

20. Yonemori K, Ueno H, Okusaka T, et al: Severe drug toxicity associated with a single-nucleotide polymorphism of the cytidine deaminase gene in a Japanese cancer patient treated with gemcitabine plus cisplatin. Clin Cancer Res 11: 26202624,2005

21. Nakahira S, Nakamori S, Tsujie M, et al: Involvement of ribonucleotide reductase M1 subunit overexpression in gemcitabine resistance of human pancreatic cancer. Int J Cancer 120 1355-1363, 2007.

22. Ohhashi S, Ohuchida K, Mizumoto K, et al: Down-regulation of deoxycytidine kinase enhances acquired resistance to gemcitabine in pancreatic cancer. Anticancer Res 28: 2205-2212, 2008.

23. Duxbury MS, Ito H, Zinner MJ, Ashley SW and Whang EE RNA interference targeting the M2 subunit of ribonucleotide reductase enhances pancreatic adenocarcinoma chemosensitivity to gemcitabine. Oncogene 23: 1539-1548, 2004

24. Kobayashi H, Murakami Y, Uemura K, Sudo T, Hashimoto Y, Kondo $\mathrm{N}$ and Sueda T: Human equilibrative nucleoside transporter 1 expression predicts survival of advanced cholangiocarcinoma patients treated with gemcitabine-based adjuvant chemotherapy after surgical resection. Ann Surg 256: 288-296, 2012.

25. Murata A, Amano R, Yamada N, Kimura K, Yashiro M, Nakata B and Hirakawa K: Prognostic predictive values of gemcitabine sensitivity-related gene products for unresectable or recurrent biliary tract cancer treated with gemcitabine alone. World J Surg Oncol 11: 117, 2013

26. Sebastiani V, Ricci F, Rubio-Viqueira B, et al: Immunohistochemical and genetic evaluation of deoxycytidine kinase in pancreatic cancer: relationship to molecular mechanisms of gemcitabine resistance and survival. Clin Cancer Res 12: 2492-2497, 2006

27. Hapke D, Stegmann A and Mitchell B: Retroviral transfer of deoxycytidine kinase into tumor cell lines enhances nucleoside toxicity. Cancer Res 56: 2343-2347, 1996.

28. Mori R, Ishikawa T, Ichikawa Y, et al: Human equilibrative nucleoside transporter 1 is associated with the chemosensitivity of gemcitabine in human pancreatic adenocarcinoma and biliary tract carcinoma cells. Oncol Rep 17: 1201-1205, 2007. 